\title{
TAp63a targeting of Lgr5 mediates colorectal cancer stem cell properties and sulforaphane inhibition
}

\author{
Yue Chen $\mathbb{1}^{1,2}$, Meng-huan Wang $\mathbb{1}^{2}$, Jian-yun Zhu $\mathbb{1}^{3}$, Chun-feng Xie $\mathbb{C}^{2}$, Xiao-ting Li $\mathbb{1}^{2}$, Jie-shu Wu $\mathbb{1}^{2}$,
} Shan-shan Geng $\mathbb{1}^{2}$, Hong-yu Han $\mathbb{1}^{4}$ and Cai-yun Zhong $\mathbb{1 0}^{2,5}$

\begin{abstract}
Cancer stem cells (CSCs) have an established role in cancer progression and therapeutic resistance. The p63 proteins are important transcription factors which belong to the p53 family, but their function and mechanism in CSCs remain elusive. Here, we investigated the role of TAp63a in colorectal CSCs and the effects of sulforaphane on TAp63a. We found that TAp63a was upregulated in spheres with stem cell properties compared to the parental cells. Overexpression of TAp63a promoted self-renewal capacity and enhanced CSC markers expression in colorectal sphere-forming cells. Furthermore, we showed that TAp63a directly bound to the promoter region of Lgr5 to enhance its expression and activate its downstream $\beta$-catenin pathway. Functional experiments revealed that sulforaphane suppressed the stemness of colorectal CSCs both in vitro and in vivo. Upregulation of TAp63a attenuated the inhibitory effect of sulforaphane on colorectal CSCS, indicating the role of TAp63a in sulforaphane suppression of the stemness in colorectal cancer. The present study elucidated for the first time that TAp63a promoted CSCs through targeting Lgr5/ $\beta$-catenin axis and participated in sulforaphane inhibition of the stem cell properties in colorectal cancer.
\end{abstract}

\section{Introduction}

Colorectal cancer remains high incidence and mortality in recent years worldwide ${ }^{1-3}$. Despite the advances in early diagnostics and interventions, colorectal cancer frequently recurs and leads to heavy burden and death ${ }^{4}$. Studies have validated the crucial roles of cancer stem cells (CSCs) in the initiation, invasion, metastasis, and drug resistance in cancer progression ${ }^{5}$. Specific markers and several signal pathways have been found to participate in the development and maintenance of colorectal $\mathrm{CSCs}^{6}$. However, the critical targets and mechanisms

Correspondence: Hong-yu Han (hanhy@sysucc.org.cn) or Cai-

yun Zhong (cyzhong@njmu.edu.cn)

'Department of Fundamental and Community Nursing, School of Nursing, Nanjing Medical University, Nanjing 211166, China

${ }^{2}$ Department of Nutrition and Food Safety, School of Public Health, Nanjing Medical University, Nanjing 211166, China

Full list of author information is available at the end of the article which contribute to colorectal CSCs have yet to be fully elucidated.

The protein p63, including multiple protein isoforms, are important transcription factors belong to the p53 family. According to the transcription start sites, the p63 gene translates to the trans-activating (TA) isoforms that contain an $\mathrm{N}$-terminal exon encoding a p53-like transactivation domain and $\Delta \mathrm{N}$ isoforms without this domain. Alternative splicing events also generate at least three isoforms, $\alpha, \beta, \gamma^{7}$. The $\alpha$ isoform is known as the predominant isoform of p63 in epithelial cells ${ }^{8}$. Based on the unique structure of each isoform, the activities of them are always elusive in different diseases. Some studies have assessed the negative relationship between TAp63 and cancer progression ${ }^{9,10}$, which implies TAp63 may inhibit CSCs. Whereas it has been reported that $\mathrm{p} 63$ promoted the functions of embryonic stem cells ${ }^{11,12}$, and TAp63 also served to maintain adult stem cells ${ }^{13}$. These studies

\section{(c) The Author(s) 2020}

(c) (i) Open Access This article is licensed under a Creative Commons Attribution 4.0 International License, which permits use, sharing, adaptation, distribution and reproduction c. in any medium or format, as long as you give appropriate credit to the original author(s) and the source, provide a link to the Creative Commons license, and indicate if changes were made. The images or other third party material in this article are included in the article's Creative Commons license, unless indicated otherwise in a credit line to the material. If material is not included in the article's Creative Commons license and your intended use is not permitted by statutory regulation or exceeds the permitted use, you will need to obtain permission directly from the copyright holder. To view a copy of this license, visit http://creativecommons.org/licenses/by/4.0/. 
suggested the promoting role of TAp63 in stem cells. The conflicting effects of TAp63 in cancer and stem cells make it an interesting topic to reveal its functions in CSCs.

G-protein-coupled receptor 5 (Lgr5), a seventransmembrane protein of the rhodopsin-like family, is recognized as a promoter of $\mathrm{Wnt} / \beta$-catenin pathway. Upon R-Spondin (RSPO) ligand binding to the receptors, Lgr5 acts in cooperation with Wnt receptors to potentiate Wnt/ $\beta$-catenin signaling ${ }^{14}$. The activation of Wnt/ $\beta$-catenin pathway also leads to the expression of Lgr5, thus serving as a feedback loop to facilitate this pathway ${ }^{15}$. Further studies confirmed the relationship of Lgr5 and colorectal CSCs, thereby establishing Lgr5 as a marker of colorectal $\mathrm{CSCs}^{16,17}$. Although the different roles of p63 on $\mathrm{Wnt} / \beta$-catenin response elements have been explored $^{18}$, the interaction between p63 and Lgr5 is not well delineated.

Epidemiological and clinical studies have shown the effectiveness of cruciferous vegetables in cancer prevention and intervention ${ }^{19,20}$. Sulforaphane (SFN) is an isothiocyanate mainly obtained from cruciferous vegetables, which is reported to be a promising anticancer agent in daily food ${ }^{21}$. Previous studies have confirmed the suppressive effect of SFN on various CSCs, including breast, pancreatic, lung $\mathrm{CSCs}^{22-24}$. Here, we examined the efficacy of SFN on colorectal CSCs in both colorectal cancer cell lines and colorectal cancer xenograft. We also investigated the regulatory role of TAp63 and Lgr5 in colorectal CSCs and in the effects of SFN on colorectal CSCs.

\section{Results}

\section{Enrichment of colorectal CSCs after sphere-forming}

The sphere-forming assay has been widely used to enrich CSCs in vitro. Here, we examined the efficiency of the enrichment after serum-free medium (SFM) treatment. As shown in Fig. 1, spheres of HCT116 and SW480 cells were generated after SFM treatment, and the sphere frequency was significantly higher than the parental serum-sustained cells (Fig. 1A, B). The mRNA and protein expressions of colorectal CSC markers, including CD133, CD44, Nanog, and Oct-4, were significantly induced compared to the parental cells maintained in

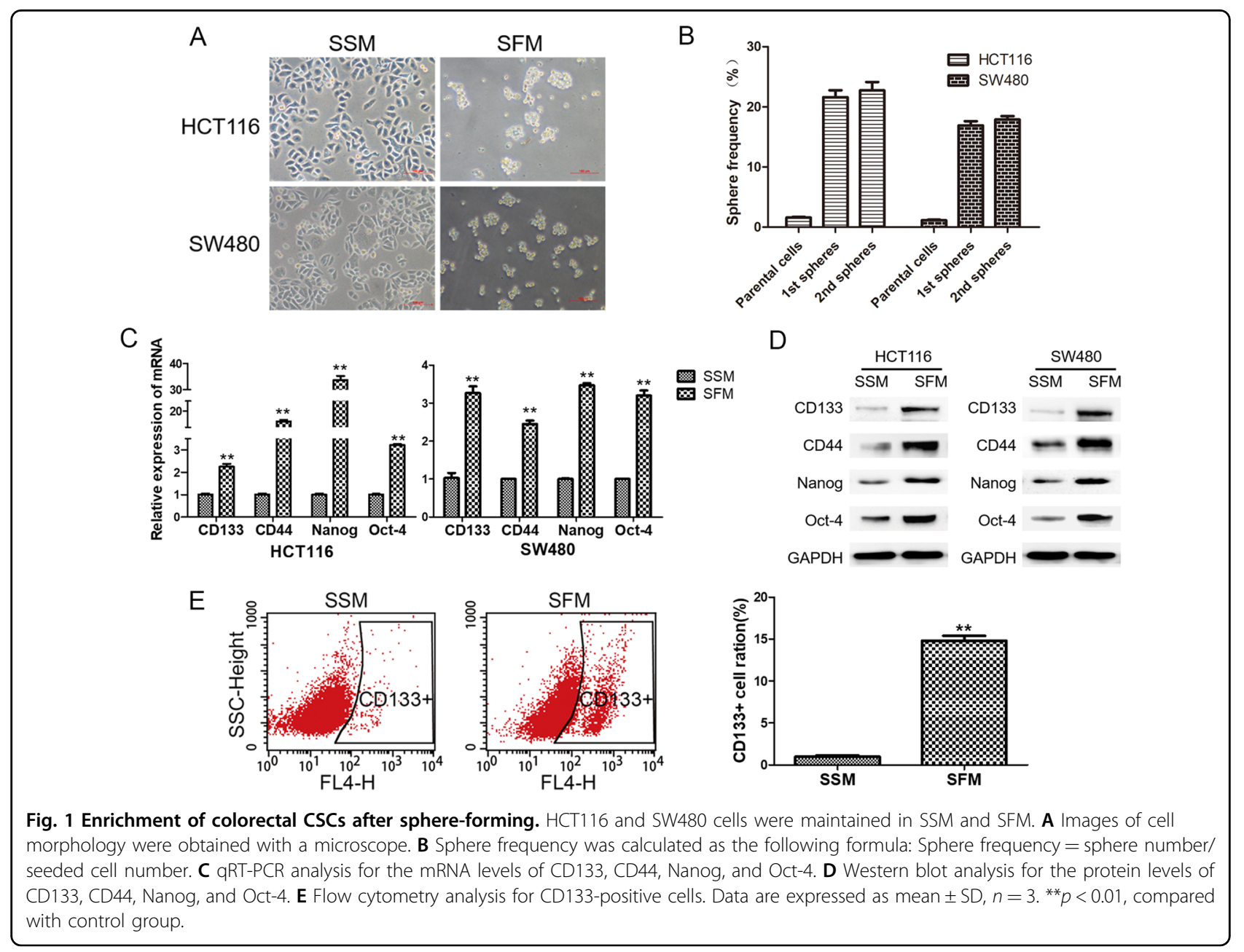


A

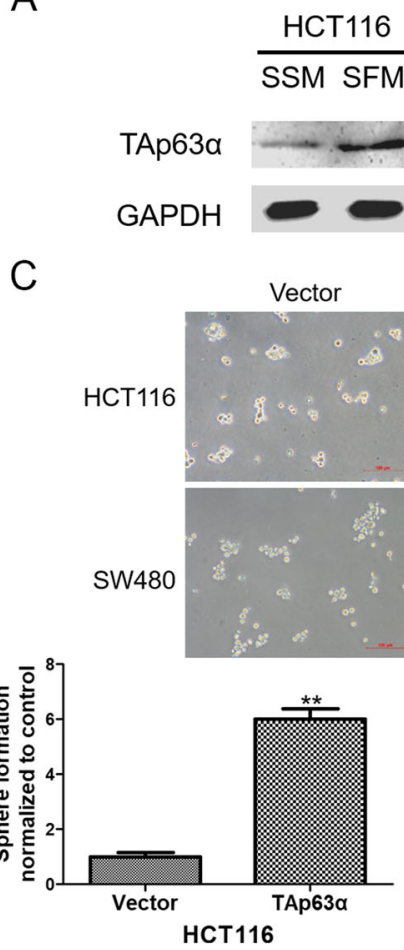

E

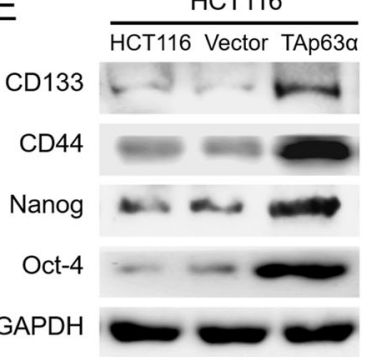

B

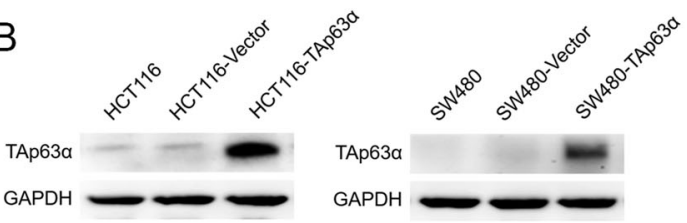

D
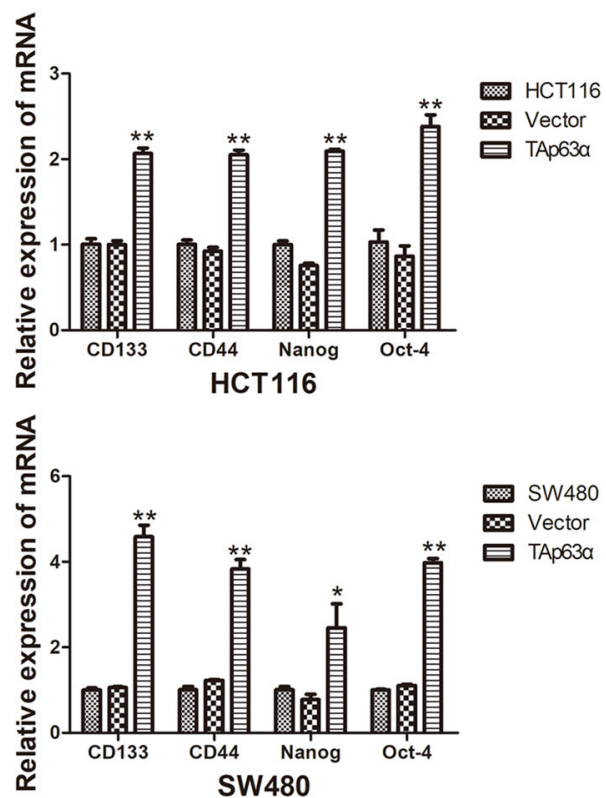

$\mathrm{F}$
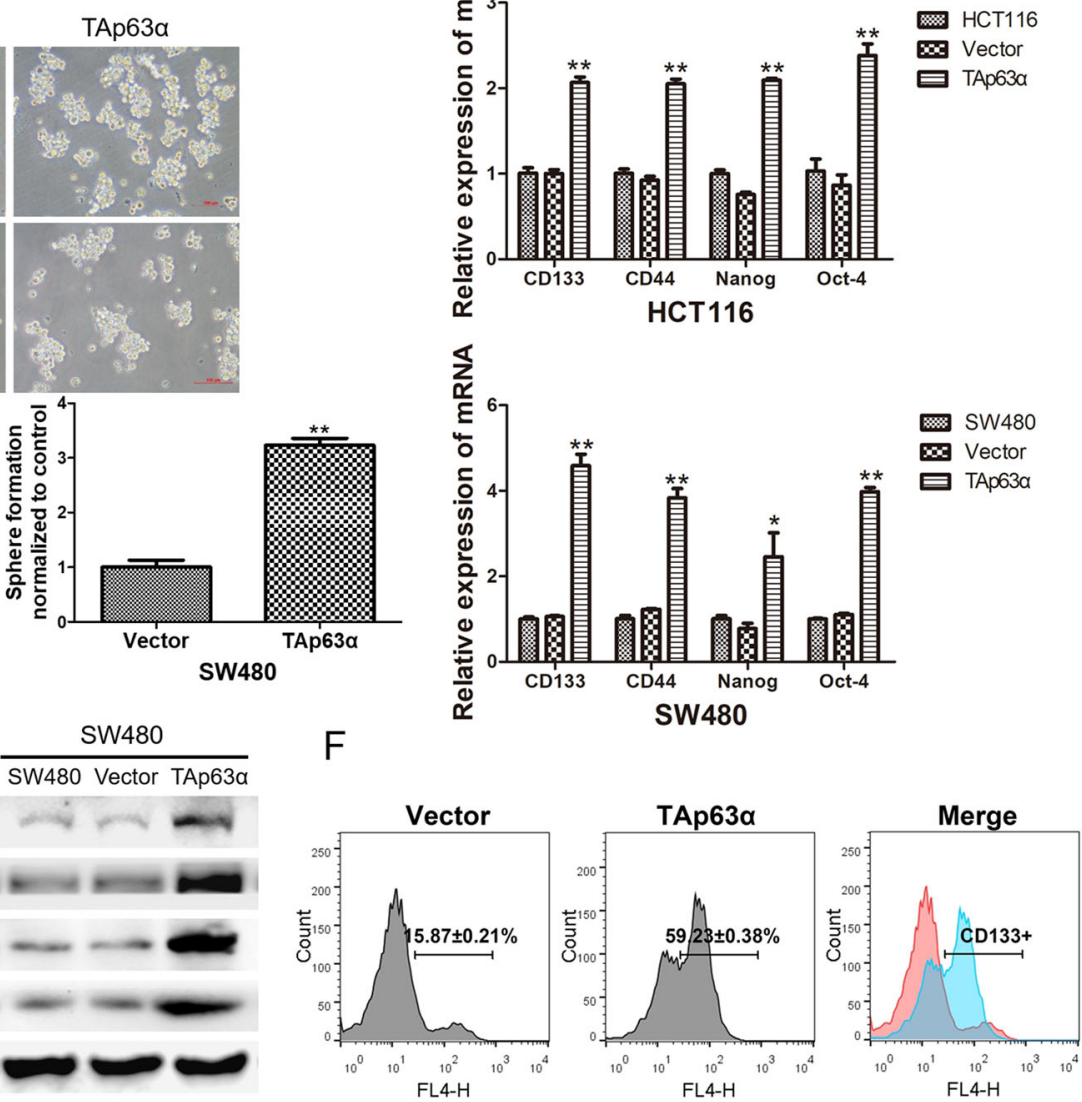

Fig. 2 TAp63a is associated with the stemness of colorectal CSCs. HCT116 and SW480 cells were used to measure the function of TAp63a. A Western blot analysis for the protein levels of TAp63a after SSM and SFM treatment. B Western blot analysis for the transfection efficiency. C Sphere-forming assay to evaluate the self-renewal ability of the cells. D qRT-PCR analysis for the mRNA levels of CD133, CD44, Nanog, and Oct-4. E Western blot analysis for the protein levels of CD133, CD44, Nanog, and Oct-4. F Flow cytometry analysis for CD133-positive cells. Data are expressed as mean $\pm \mathrm{SD}, n=3 .{ }^{*} p<0.05$ and ${ }^{* *} p<0.01$, compared with control group.

serum-sustained medium (SSM) (Fig. 1C, D). Flow cytometry analysis showed about $15 \%$ increase of CD133positive cells $(p<0.05)$ after sphere-forming (Fig. 1E). These results suggested the characteristics of CSCs in HCT116 and SW480 sphere-forming cells cultured in SFM.

\section{TAp63a is associated with the stemness of colorectal CSCs}

The preceding data prompted us to assess the expression of p63 in sphere-forming cells, and we found elevated expression levels of TAp63 $\alpha$ in the spheres (Fig. 2A). To further determine the role of TAp63 $\alpha$ in colorectal CSCs, we constructed a plasmid to elevate the expression of TAp63 $\alpha$ in HCT116 and SW480 cells (Fig. 2B). The sphere-forming assay was applied to measure the selfrenewal property of CSCs and showed that TAp63 $\alpha$ facilitated the sphere formation ability of colorectal cancer cells. As shown in Fig. $2 \mathrm{C}$, the size of the spheres was increased, and the number of the spheres was enhanced up to 6 -fold $(p<0.05)$. We next examined the expression of mRNA and protein levels of colorectal CSC markers and found that overexpression of TAp63 $\alpha$ upregulated the mRNA and protein levels of these markers (Fig. 2D, E). Flow cytometry analysis also showed that TAp63 $\alpha$ transfection led to about 4-fold increase $(p<0.05)$ of CD133-positive cells compared to vector transfection group after sphere formation (Fig. 2F). These results 

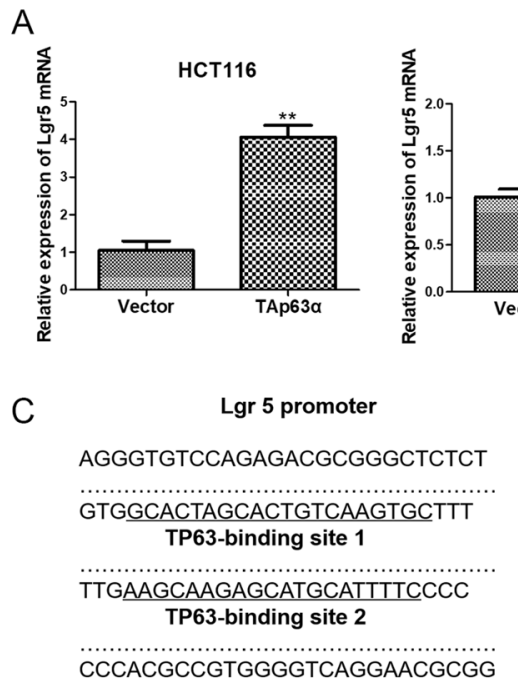

B
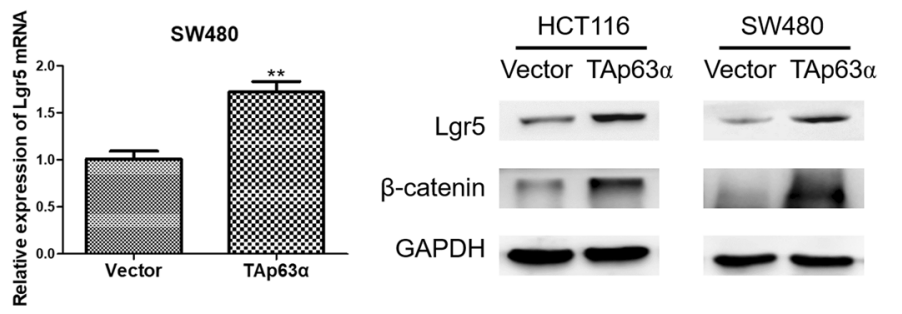

D
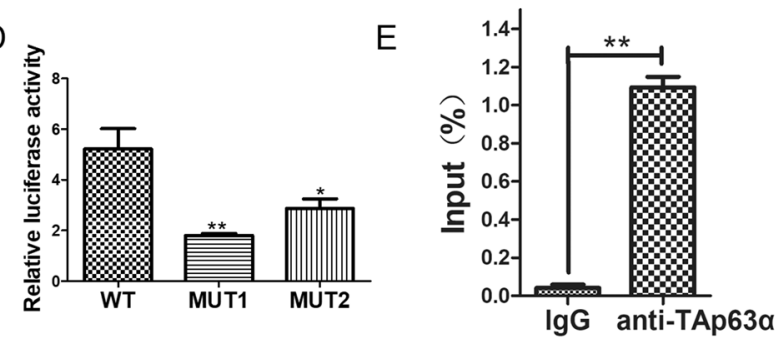
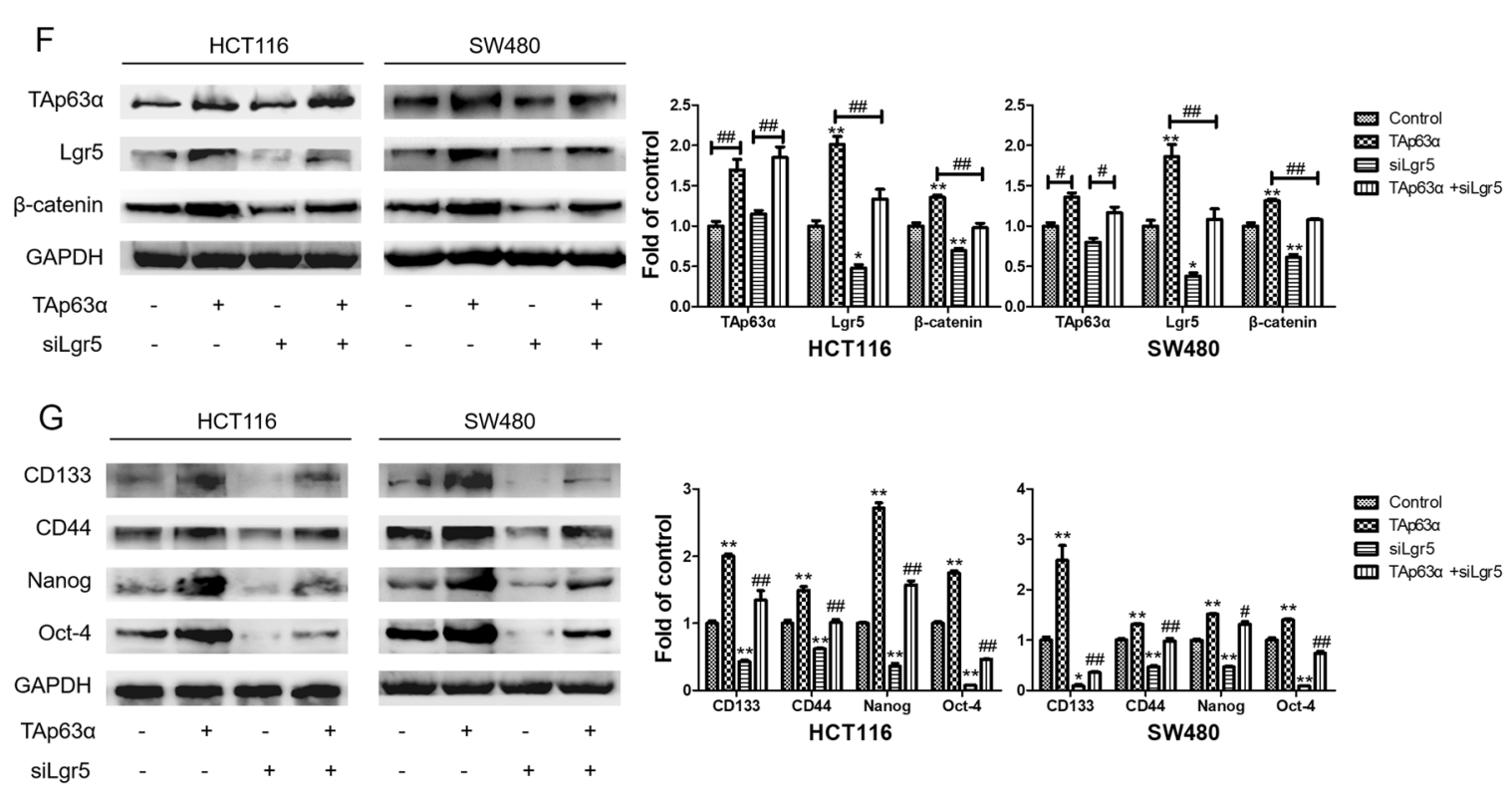

Fig. 3 TAp63a directly targets Lgr5 to promote stemness in colorectal CSCs. HCT116 and SW480 cells were used to measure the regulation role of TAp63a on Lgr5. A qRT-PCR analysis for the mRNA levels of Lgr5 transfection. Data are expressed as mean $\pm S D, n=3$. ${ }^{* *} p<0.01$, compared with vector group. B Western blot analysis for the protein levels of Lgr5 and $\beta$-catenin. C Two binding sites of TP63 in the Lgr5 promoter region. D Dualluciferase reporter assay for the transcriptional activity of Lgr5. Fold changes of the luciferase activity were relative to the vector group. Data are expressed as mean $\pm \mathrm{SD}, n=3 .{ }^{*} p<0.05$ and ${ }^{*} p<0.01$, compared with wild-type (WT) group. E ChIP assay for the binding capacity of TAp63a in Lgr5 promoter region after TAp63a was overexpressed. F Western blot and densitometric analyses for the protein levels of TAp63a, Lgr5, and $\beta$-catenin. Data are expressed as mean $\pm \mathrm{SD}, n=3 .{ }^{*} p<0.05,{ }^{* *} p<0.01$, compared with control group. ${ }^{\#} p<0.05$, ${ }^{\# \#} p<0.01$. G Western blot and densitometric analyses for the protein levels of CD133, CD44, Nanog, and Oct-4. Data are expressed as mean \pm SD, $n=3 .{ }^{*} p<0.05,{ }^{* *} p<0.01$, compared with control group. ${ }^{*} p<0.05,{ }^{\# \#} p<0.01$, compared with TAp63a group.

suggested that TAp63 $\alpha$ promoted the stem cell-like properties in colorectal cancer cells.

\section{TAp63a directly targets Lgr5 to promote stemness in colorectal CSCs}

After TAp63 $\alpha$ plasmid transfection, we observed upregulated mRNA and protein levels of Lgr5 in colorectal cancer cells (Fig. 3A, B). The immunoblotting analysis further confirmed the downstream $\beta$-catenin accumulation upon Lgr5 elevation (Fig. 3B). Bioinformatics method was then applied to show that there were two binding sites of TP63 in Lgr5 promoter region, and thus indicated the transcriptional regulation of TAp63 $\alpha$ on Lgr5 (Fig. 3C). To identify the direct binding site of TAp63 $\alpha$, we 
constructed the wild-type luciferase reporter plasmid and the corresponding mutants. Dual-luciferase reporter assay showed that overexpression of TAp63 $\alpha$ increased the transcriptional activity of Lgr5, while the luciferase activity was decreased with the mutation of the binding sites (Fig. 3D). Besides, ChIP assay confirmed the binding capacity of TAp63 $\alpha$ in Lgr5 promoter region when TAp63 $\alpha$ was overexpressed (Fig. 3E). Moreover, knockdown of Lgr5 with siRNA attenuated $\beta$-catenin accumulation and colorectal CSC markers expression triggered by TAp63 $\alpha$ overexpression (Fig. 3F, G). Collectively, these findings illustrated that TAp63 $\alpha$ directly targets Lgr5 at transcriptional level to promote colorectal CSCs.

\section{SFN diminishes CSC-like properties of colorectal cancer in vitro}

We then examined the effects of SFN on colorectal CSCs. As shown in Fig. 4, the size and the number of the colorectal cancer spheroids were decreased in a dose-dependent manner after the treatment of the spheroids with different concentrations of SFN for 4 days (Fig. 4A). Immunoblotting analysis showed that SFN treatment also led to the downregulation of the colorectal CSC markers, including CD133, CD44, Nanog, and Oct-4 (Fig. 4B). Besides, the percentage of CD133-positive cells was diminished from $13.39 \%$ to $4.25 \%$ in spheroids treated with $10 \mu \mathrm{M}$ of SFN for 4 days (Fig. 4C). Furthermore, colony formation assay showed that SFN suppressed the formation of colonies in both cell spheroids (Fig. 4D), and CCK-8 assay confirmed the decline of cell viability in the spheroids (Fig. 4E). Overall, these results suggested that SFN inhibited colorectal CSC properties of HCT116 and SW480 cell spheroids in vitro.

\section{TAp63a mediates SFN-suppressed CSC-like properties}

Considering the critical role of TAp63 $\alpha / \mathrm{Lgr} 5 / \beta$-catenin axis in colorectal CSCs, we examined the effects of SFN on TAp63 $\alpha$ and its downstream Lgr5 and $\beta$-catenin. As shown in Fig. 5A, concomitant with the repression of CSC properties, SFN treatment also led to downregulation of TAp63 $\alpha$ in HCT116 and SW480 cell spheroids. Using sphereforming assay, we verified that the inhibition of SFN on CSC self-renewal ability was reversed by TAp63 $\alpha$ overexpression (Fig. 5B). Results of immunoblotting analysis further showed that SFN-induced Lgr5 and $\beta$-catenin suppression can be alleviated by TAp63 $\alpha$ overexpression (Fig. 5C). Likewise, the repression of SFN on colorectal CSC markers was also diminished after TAp63 $\alpha$ transfection (Fig. 5D, E). Together, these data suggested that TAp63 $\alpha$ mediated the inhibitory effects of SFN on CSC properties in colorectal cancer.

\section{SFN inhibits TAp63a/Lgr5 axis and CSC-like properties in vivo}

To investigate the effects of SFN on colorectal CSCs in vivo, HCT116 colorectal cancer xenograft model was established in nude mice. After injected subcutaneously with cells in the front dorsum, mice were divided into three groups and treated with SFN or $0.9 \%$ sodium chloride solution through intraperitoneal injection every 3 days (Fig. 6A). As shown in Fig. 6B, C, SFN treatment resulted in a significant decrease in tumor size and weight $(p<0.05)$. Immunohistochemistry staining validated the inhibition in tumor cell proliferation (Fig. 6D). As expected, the expression of colorectal CSC markers, including CD133, CD44, Nanog, and Oct-4, also decreased in the xenograft after SFN treatment (Fig. 6E). In concert with these colorectal CSC markers, inhibition of TAp63 $\alpha /$ Lgr5/ $\beta$-catenin axis was also observed (Fig. 6F). Collectively, these in vivo data regarding SFN effects on TAp63 $\alpha$ activation and colorectal CSCs were consistent with in vitro results, suggesting the role of TAp63 $\alpha / \operatorname{Lgr} 5 /$ $\beta$-catenin axis in this process.

\section{Discussion}

Several reports suggested the suppressive role of TAp63 in cancer progression. However, studies focused on stem cells showed that TAp63 promote the stem cell properties in embryonic and adult stem cells. Here, we investigated the function of TAp63 $\alpha$, one of the important p63 isoforms in epithelial cells, in colorectal CSCs, and showed that TAp63 $\alpha$ directly targets Lgr $5 / \beta$-catenin axis to promote stem cell properties in colorectal CSCs. Besides, we found that SFN inhibits colorectal CSC properties via TAp63 $\alpha /$ Lgr5/ $\beta$-catenin axis suppression.

The p63 family members are crucial regulators of cancer and have been found to play an important role in embryonic stem cells ${ }^{25-27}$. As reported by several studies, TAp63 suppressed the progression of cancers, and thus be considered as a promising strategy for therapeutics ${ }^{9,10,28}$. However, other studies have shown that TAp63 also promoted stem cell maintenance and differentiation ${ }^{12,13}$. Hence, the function of TAp63 in CSCs has attracted attention. The diverse isoforms of TAp63 may have different functions; while this study focused on $\alpha$ isoform because it is the predominant isoform of TAp63 in epithelial cells. We found that upregulation of TAp63 $\alpha$ led to elevated self-renewal ability and CSC markers expression in colorectal cancer. These data indicated the activating role of TAp63a on colorectal CSCs, which support the previous studies in embryonic and adult stem cells, thus suggesting that TAp63 $\alpha$ may be considered as a target of colorectal CSCs. To further explore the downstream genes of TAp63 $\alpha$ in CSCs, we used bioinformatics approach and found two binding sites of TP63 on Lgr5 promoter. Results from dual-luciferase reporter and ChIP assay confirmed the existence and activity of the TAp63 $\alpha$ binding complex. Moreover, the effects of TAp63 $\alpha$ on Lgr $5 / \beta$-catenin axis and colorectal CSC markers were also attenuated by Lgr5 silencing. Together, these results 
A

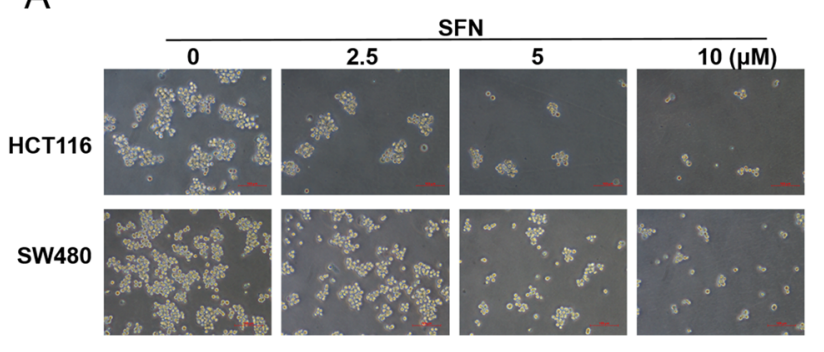

B

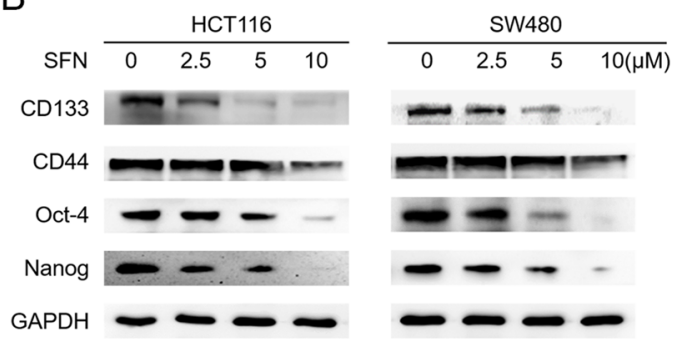

C

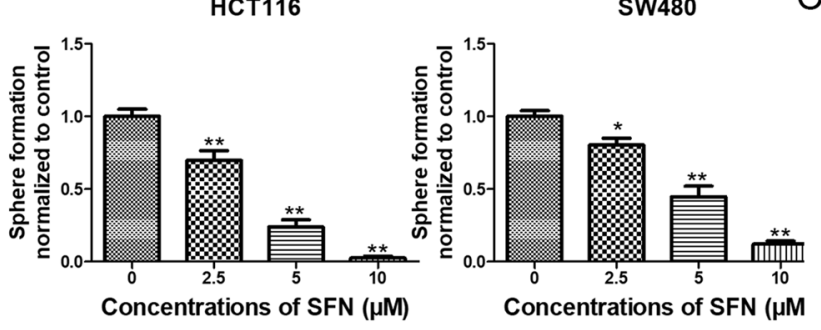

D

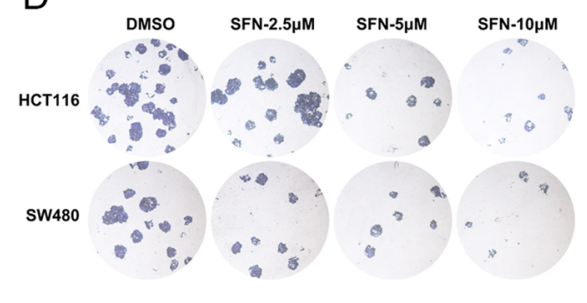

E
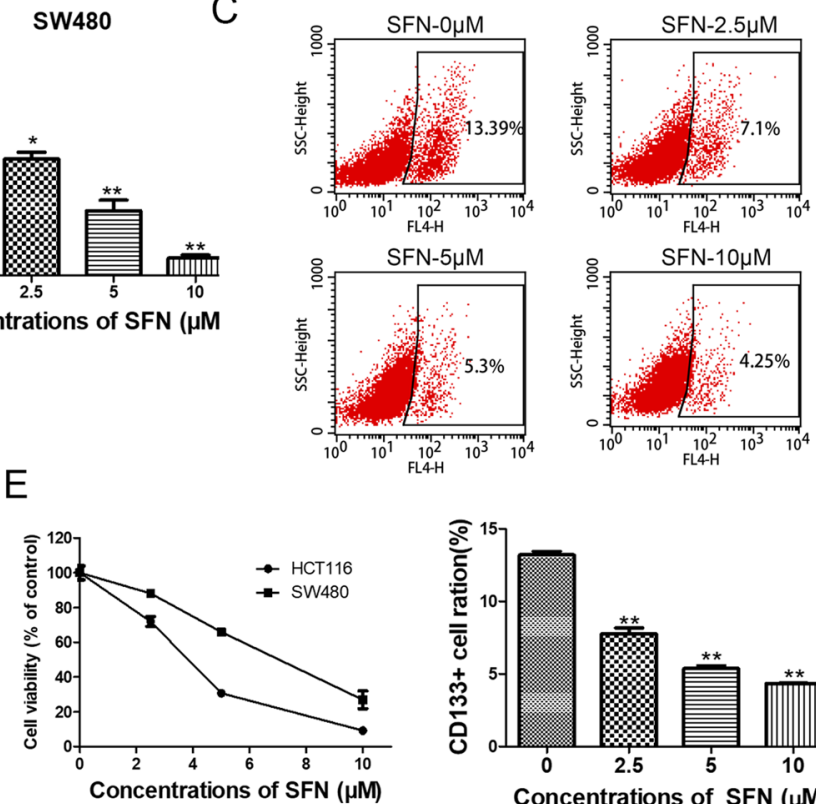

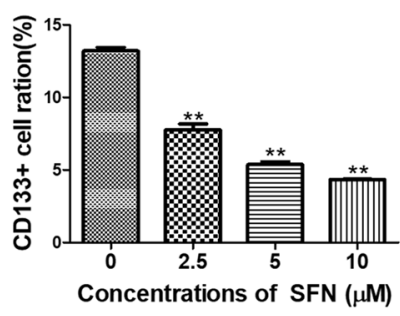

Fig. 4 SFN diminishes CSC-like properties of colorectal cancer in vitro. HCT116 and SW480 spheres were treated with different concentrations of SFN for 4 days, and $0.1 \%$ DMSO was used as solvent control. A Sphere-forming assay was used to determine the size and number of spheres. B Western blot analysis for the protein levels of CD133, CD44, Nanog, and Oct-4. C Flow cytometry analysis for CD133-positive cells. D Colony formation of the spheres after SFN treatment. E Cell viability was measured by CCK-8 assay after SFN treatment of cell spheroids. Data are expressed as mean $\pm \mathrm{SD}, n=3 .{ }^{*} p<0.05$ and ${ }^{* *} p<0.01$, compared with control group.

suggested that TAp63 $\alpha$ directly targets Lgr5 to promote stemness in colorectal CSCs.

Since mounting evidence has established CSCs as the driving force in cancer progression, great efforts have been made to explore new strategies targeting CSCs. $\mathrm{Wnt} / \beta$-catenin pathway is widely recognized as a crucial regulator of colorectal CSCs, and Lgr5 is a component of this pathway to potentiate Wnt signal and eventually leads to the activation of $\beta$-catenin. Besides, Lgr5 is now widely utilized as a stem cell marker in gastrointestinal cancers $^{29,30}$, which indicates the role of Lgr5 in CSC properties. Here, we observed significant inhibition of CSC markers after Lgr5 silence, which suggested that Lgr5 may not only be used as a marker but also served as a target in colorectal CSCs.

In the past years, studies have reported the tumorigenesis role of $\Delta \mathrm{Np} 63$, the critical $\Delta \mathrm{N}$ isoforms of p63 expressed in epithelial tissues. It was shown that $\Delta \mathrm{Np} 63$ promoted self-renewal and breast tumor growth through sonic hedgehog pathway in mammary $\mathrm{CSCs}^{7}$. Meanwhile, $\Delta \mathrm{Np} 63 \alpha$ was revealed as an oncogene for cooperating with Ras to promote tumor-initiating stem-like cells in skin stem cells ${ }^{31}$. These previous studies depicted the relationship between $\Delta \mathrm{Np} 63$ and CSC properties. Although the present study focused on the role of TAp63 $\alpha$ in colorectal CSCs, it is worth noting that further studies to ascertain the tumorigenic role of $\triangle \mathrm{Np} 63$ in different cellular context, including colorectal CSCs, are necessary.

SFN is a natural product mainly obtained from cruciferous vegetables with anticancer activities ${ }^{32}$. Previous studies found that SFN exerted its anticancer effects through the regulation of drug-metabolizing enzymes ${ }^{21,33}$. Subsequent studies found that SFN also suppressed cell 


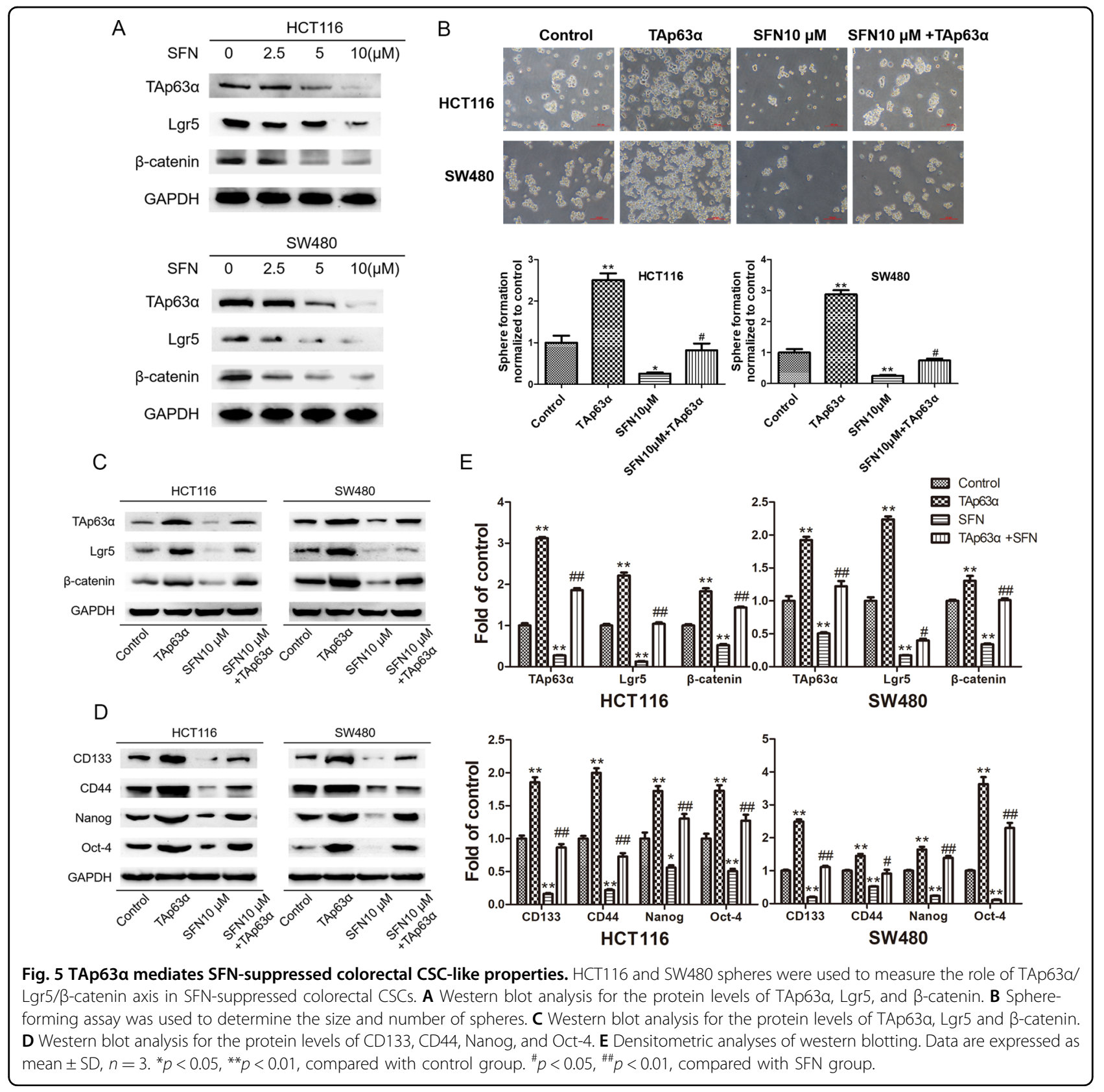

cycle and metastasis of cancer cells ${ }^{20,34}$. Recently, the inhibitory effects of SFN on CSCs have been reported ${ }^{22-24}$. However, the underlying targets by which SFN inhibited colorectal CSCs have yet to be fully elucidated. Here, we confirmed the inhibitory effects of SFN on colorectal CSCs both in vitro and in vivo, and found that SFN inhibited colorectal CSCs properties through TAp63 $\alpha$ / Lgr5/ $\beta$-catenin axis.

Although certain variables, such as the site of implantation, the properties of cell lines, and the growth properties of the xenograft, can affect the stability and reproducibility of xenograft formation ${ }^{35,36}$, cell line- derived xenograft models have been widely used in cancer research attribute to its high accessibility in laboratory settings ${ }^{37}$. Here, based on the results of our preliminary experiments, HCT116 cells were chosen to produce stable xenograft in mice in our system. It should be noted that although the in vitro results showed the promotion role of TAp63 $\alpha$ on colorectal CSCs, future studies are warranted to explore the function of TAp63 $\alpha$ in both in vitro and in vivo models.

In summary, we illustrated for the first time that TAp63 $\alpha$ directly regulated Lgr5 expression to promote colorectal CSCs. Findings from this study suggested 


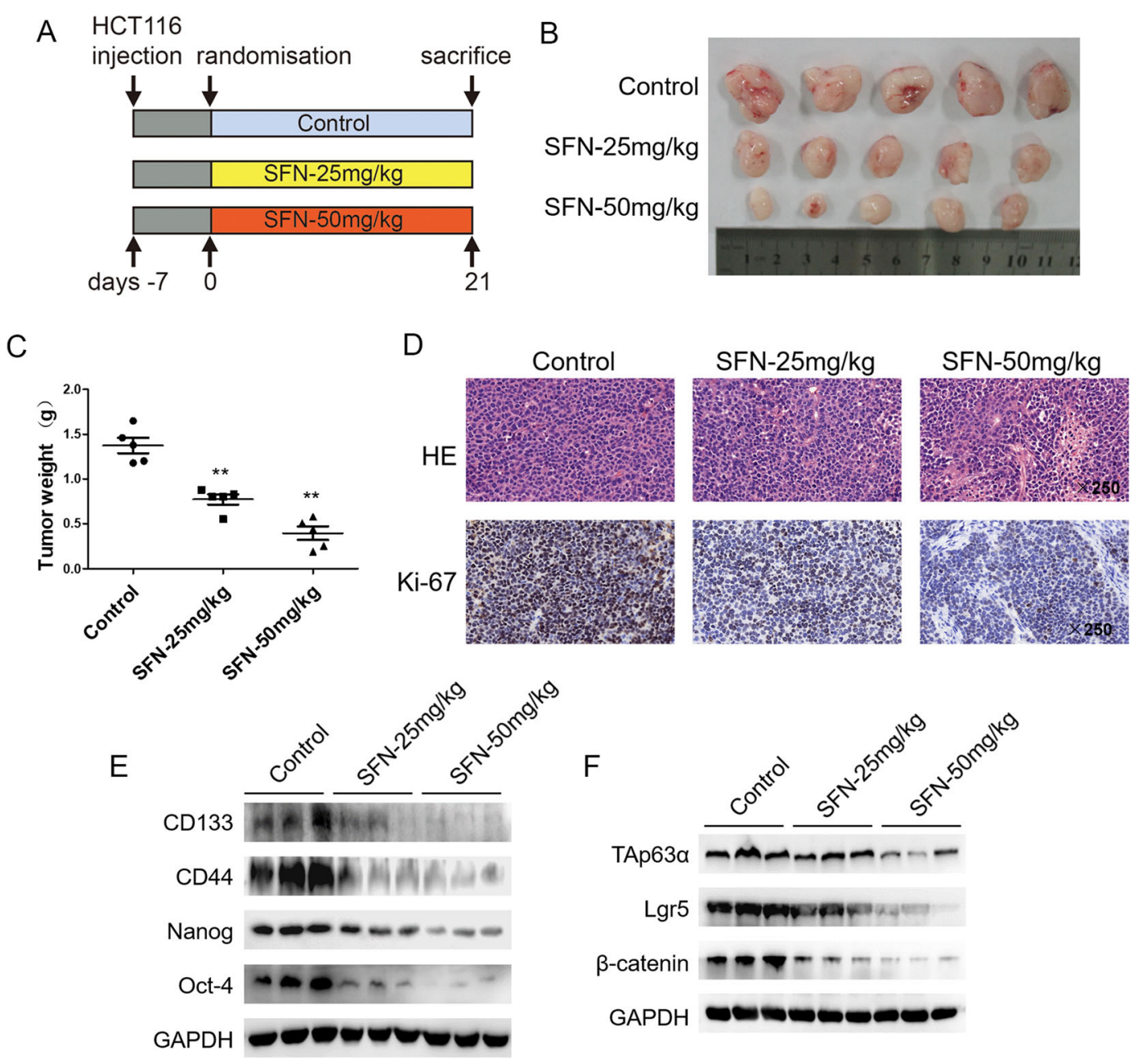

Fig. 6 SFN inhibits TAp63a/Lgr5 axis and CSC-like properties in vivo. HCT116 cells were injected subcutaneously in the front dorsum of nude mice. A Mice were divided into three groups and treated with SFN or $0.9 \%$ sodium chloride solution. B Tumor volume after SFN treatment. C Tumor weight after SFN treatment. Data are expressed as mean $\pm \mathrm{SD}, n=3 .{ }^{*} p<0.01$, compared with control group. $\mathbf{D} H \& \mathrm{E}$ and $\mathrm{IHC}$ staining for the xenograft. E Western blot analysis for the protein levels of CD133, CD44, Nanog, and Oct-4. F Western blot analysis for the protein levels of TAp63a, Lgr5, and $\beta$-catenin.

therapeutic potential of SFN in the treatment of colorectal cancer through inhibiting colorectal CSCs via targeting TAp63 $\alpha /$ Lgr5/ $\beta$-catenin axis.

\section{Materials and methods}

\section{Cell culture and reagents}

Human colon cancer cell lines HCT116 and SW480 were obtained from Shanghai Institute of Cell Biology, Chinese Academy of Sciences (Shanghai, China) and maintained in RPMI 1640 medium (Gibco, Carlsbad, CA, USA) supplemented with $10 \%$ fetal bovine (Gibco, Carlsbad, CA, USA), 100 units $/ \mathrm{mL}$ penicillin and $100 \mathrm{mg} /$ $\mathrm{mL}$ streptomycin (Gibco, Carlsbad, CA, USA) at $37^{\circ} \mathrm{C}$ in incubator containing $5 \% \mathrm{CO}_{2}$. SFN and dimethyl sulfoxide (DMSO) were purchased from Sigma (St. Louis, MO, USA).

\section{Sphere-forming assay}

Cells were seeded in non-adherent dishes (Costar, NY, USA) in the incubator with $5 \% \mathrm{CO}_{2}$ at $37^{\circ} \mathrm{C}$ and maintained in serum-free medium (SFM) composed of DMEM-F12 (Gibco, Carlsbad, CA, USA), $20 \mathrm{ng} / \mathrm{mL}$ epidermal growth factor (Rocky Hill, NJ, USA), $10 \mathrm{ng} / \mathrm{mL}$ basic fibroblast growth factor (Rocky Hill, NJ, USA), $5 \mu \mathrm{g} /$ $\mathrm{mL}$ insulin (Rocky Hill, NJ, USA), 0.4\% BSA (Sigma, St. Louis, MO, USA), 2\% B27 (Gibco, Carlsbad, CA, USA). The pictures and numbers of spheres were photographed under a microscope (Nikon, Tokyo, Japan) and counted if the diameter was greater than $50 \mu \mathrm{m}$. Colorectal cancer cells, 1st and 2nd spheres were seeded in 24-well plates $(10,000$ cells/well), and spheres were counted after 6 days. Sphere frequency was calculated as the following formula: Sphere frequency $=$ sphere number/10,000. Spheroids 
viability was assessed by cell counting kit-8 (CCK-8) (Beyotime, Shanghai, China) according to the manufacturer's instructions.

\section{Flow cytometry}

For the detection of CD133-positive cells, cells were stained with CD133 antibody (Miltenyi Biotec, Teterow, Germany) at $4{ }^{\circ} \mathrm{C}$ for $10 \mathrm{~min}$ in the dark. Immunoglobulin G (IgG) isotype staining (Miltenyi Biotec, Teterow, Germany) was used as a negative control. Cell strainers were used to separate single cells before detection. The samples were analyzed using the FACS ArialII system (BectonDickinson, San Jose, CA, USA) and the results were analyzed using FlowJo software (Ashland, OR, USA).

\section{Immunoblotting analysis}

Immunoblotting analysis was performed as described previously $^{38}$. In brief, $40 \mu \mathrm{g}$ of total proteins in $15 \mu \mathrm{L}$ were subject to 7.5-10\% SDS-PAGE, transferred to nitrocellulose filter membrane (Millipore, Billerica, MA, USA), exposed to the antibodies, and detected by Chemiluminescence western blotting reagents (Cell Signaling Technology, Danvers, MA). GAPDH was served as the loading control. Antibodies used in the present study were purchased from Proteintech (Rocky Hill, NJ, USA).

\section{Quantitative real-time polymerase chain reaction (qRT- PCR)}

Total RNA was isolated with Trizol reagent (Invitrogen, Carlsbad, CA, USA). The cDNA was synthesized with 5×All-In-One RT MasterMix (Applied Biosystems, Foster City, CA, USA). The EvaGreen $2 \times q \mathrm{PCR}$ MasterMix (Applied Biosystems, Foster City, CA, USA) and LC96 real-time PCR system were applied for detection. The PCR primers were purchased from Beijing Genomics Institute (Beijing, China). Data were standardized by GAPDH. Primer sequences are listed as follows:

GAPDH-F, 5'-CAAGGTCACCATGACAACTTTG-3'; GAPDH-R, 5'-GTCCACCACCCTGTTGCTGTAG-3'; CD44-F, 5'-AGGATTTCCCCAGAACTTAG-3'; CD44-R, 5'-ACAGGTCAAGATGGAAGATG-3'; CD133-F, 5'-GCACTCTATACCAAAGCGTCAA-3'; CD133-R, 5'-CTCCCATACTTCTTAGTTTCCTCA-3'; Nanog-F, 5'-AGAAGGCCTCAGCACCTA-3'; Nanog-R, 5'-GGCCTGATTGTTCCAGGATT-3'; Oct4-F, 5'-ACATCAAAGCTCTGCAGAAAGAACT-3'; Oct4-R, 5'-CTGAATACCTTCCCAAATAGAACCC-3'; Lgr5-F, 5'-GAGTTACGTCTTGCGGGAAAC-3'; Lgr5-R, 5'-TGGGTACGTGTCTTAGCTGATTA-3'.

\section{Transient transfection}

HCT116 and SW480 cells were cultured in RPMI 1640 cell culture medium and seeded in six-well plates at a density of $2 \times 10^{5}$ cells. Human pcDNA3.1-TAp63 $\alpha$ and vector plasmids were purchased from Addgene (Rockville, USA). Human Lgr5 or control small-interfering RNA (siRNA) were purchased from RiBoBio (Guangzhou, China). Plasmids or siRNA were transfected into cells using the Lipofectamine 3000 reagent (Invitrogen, Carlsbad, CA, USA) following the manufacturer's protocol. After transfections, cells were cultured in RPMI 1640 medium supplemented with $10 \%$ fetal bovine serum (Gibco, Carlsbad, CA, USA) before using for other experiments.

\section{Dual-luciferase reporter assay}

The luciferase reporter plasmids contain the wild-type or mutant Lgr5 promoter into the pGL3 vector were generated by Genechem (Shanghai, China). Cells were seeded in 24-well plates and cultured $24 \mathrm{~h}$ before transfection. pGL3 vector, Lgr5-WT, Lgr5-MUT1 or Lgr5MUT2 were co-transfected with pcDNA3.1-TAp63 $\alpha$ and pRL-SV40 with lipofectamine 3000 reagent (Invitrogen, Carlsbad, CA, USA). Luciferase and Renilla activities were measured $48 \mathrm{~h}$ after transfection with the dual-luciferase reporter assay kit (Promega, Madison, WI, USA) following the manufacturer's introductions. Renilla luciferase activities were used for normalization.

\section{Chromatin immunoprecipitation (ChIP)}

ChIP assays were performed with chromatin immunoprecipitation kit purchased from Cell Signaling Technology (Danvers, MA, USA) in accordance with the manufacturer's procedure. Anti-TAp63 $\alpha$ and normal IgG antibodies were used to precipitate the DNA-protein complexes. Precipitated DNA was amplified with Lgr5 promoter-specific primers: 5'-TACTGATTGTGCGGA AAC-3' (forward) and $5^{\prime}$-TGGAGAAAGTCGTCGAG $T-3^{\prime}$ (reverse). The PCR products were analyzed by qRT-P $\mathrm{CR}$ analysis.

\section{Colony formation assay}

Cell spheroids were separated into single cells and then seeded into cell culture dishes (Costar, NY, USA) maintained in RPMI 1640 medium at $37^{\circ} \mathrm{C}$ in an incubator containing $5 \% \mathrm{CO}_{2}$ for 10 days after treatment. Colonies were fixed in $4 \%$ paraformaldehyde solution for $10 \mathrm{~min}$ and stained with crystal violet for $10 \mathrm{~min}$. After been washed with $\mathrm{PBS}$, the colonies were then photographed and counted under a microscope (Olympus, Tokyo, Japan).

\section{In vivo experiments}

For in vivo studies, female BALB/c nude mice (4 weeks old, $20 \mathrm{~g}$ ) were purchased from Shanghai Animal Laboratory Center and maintained in the Experimental Animal Center at Nanjing Medical University with appropriate sterile filter-capped cages. All animal 
experiments were conducted under the instructions of the Guide for the Care and Use of Laboratory Animals of the National Institutes of Health strictly. The protocol was approved by the Committee on the Ethics of Animal Experiments of Nanjing Medical University (IACUC1802010). HCT116 cells $\left(5 \times 10^{6}\right.$ each) were injected subcutaneously in the front dorsum. At 7 days posttransplantation, the xenografts were randomized into three groups (five mice in each group). SFN or $0.9 \%$ sodium chloride solution were given through intraperitoneal injection every 3 days. No blinding was conducted during the experiments. The tumor volumes were calculated as the following formula: volume $\left(\mathrm{mm}^{3}\right)=$ length $\times$ width $\times$ width $/ 2$. After 4 weeks of treatment, the mice were sacrificed, and the xenografts were removed for examination.

\section{Tissue immunohistochemistry staining}

Tumor xenografts were fixed in $4 \%$ paraformaldehyde solution before paraffin embedding. Hematoxylin and eosin (H\&E) and IHC staining were performed by the Servicebio (Wuhan, China). Briefly, paraffin-embedded sections were deparaffinized and hydrated in xylene, ethanol, and water. Sections were incubated with primary antibody at $4{ }^{\circ} \mathrm{C}$ overnight, followed by the corresponding secondary antibodies and developed with diaminobenzidine. The slides were counterstained with haematoxylin and mounted in xylene mounting medium for examination. Pannoramic scanner system was used to observe the cell images.

\section{Statistical analysis}

All data are expressed as mean \pm SD. Student's $t$ test was used to compare the difference between two groups. Oneway analysis of variance (ANOVA) with Dunnet or Bonferroni post hoc test was used for multiple comparisons. Significant difference was taken as ${ }^{*} p<0.05$ or ${ }^{* *} p<0.01$ or $\# p<0.05$. All analyses were performed with SPSS version 11.0 software.

\section{Acknowledgements}

This work was supported by grants from the National Natural Science Foundation of China (No. 81773431, No. 81973026) and the Postgraduate Research \& Practice Innovation Program of Jiangsu Province (JX22013548).

\footnotetext{
Author details

'Department of Fundamental and Community Nursing, School of Nursing, Nanjing Medical University, Nanjing 211166, China. ${ }^{2}$ Department of Nutrition and Food Safety, School of Public Health, Nanjing Medical University, Nanjing 211166, China. ${ }^{3}$ Suzhou Digestive Diseases and Nutrition Research Center, Suzhou Municipal Hospital, The Affiliated Suzhou Hospital of Nanjing Medical University, Suzhou 215000, China. ${ }^{4}$ Department of Clinical Nutrition, Sun Yatsen University Cancer Center, State Key Laboratory of Oncology in South China, Collaborative Innovation Center for Cancer Medicine, Guangzhou 510060, China. ${ }^{5}$ Center for Global Health, School of Public Health, Nanjing Medical University, Nanjing 211166, China
}

Conflict of interest

The authors declare that they have no conflict of interest.

\section{Publisher's note}

Springer Nature remains neutral with regard to jurisdictional claims in published maps and institutional affiliations.

Received: 6 December 2019 Revised: 22 September 2020 Accepted: 25 September 2020

Published online: 10 October 2020

\section{References}

1. Bray, F. et al. Global cancer statistics 2018: GLOBOCAN estimates of incidence and mortality worldwide for 36 cancers in 185 countries. CA Cancer J. Clin. 394-424 (2018).

2. Siegel, R. L., Miller, K. D. \& Jemal, A. Cancer statistics, 2019. Ca-Cancer J. Clin. 69 7-34 (2019).

3. Ferlay, J. et al. Cancer incidence and mortality patterns in Europe: estimates for 40 countries and 25 major cancers in 2018. Eur. J. Cancer 103, 356-387 (2018).

4. Cunningham, D. et al. Colorectal cancer. Lancet 375, 1030-1047 (2010).

5. Ayob, A. Z. \& Ramasamy, T. S. Cancer stem cells as key drivers of tumour progression. J Biomed Sci. 25, 20. https://doi.org/10.1186/s12929-018-0426-4 (2018).

6. Zeuner, A., Todaro, M., Stassi, G. \& De Maria, R. Colorectal cancer stem cells: from the crypt to the clinic. Cell. Stem Cell. 15, 692-705 (2014).

7. Memmi, E. M. et al. p63 sustains self-renewal of mammary cancer stem cells through regulation of Sonic Hedgehog signaling. Proc. Natl Acad. Sci. USA 112, 3499-3504 (2015).

8. Crum, C. P. \& McKeon, F. D. p63 in epithelial survival, germ cell surveillance, and neoplasia. Ann. Rev. Phytopathol. 5, 349 (2010).

9. Lin, C. W. et al. TAp63 suppress metastasis via miR-133b in colon cancer cells. Brit J. Cancer 110, 2310-2320 (2014).

10. Su, X. et al. TAp63 suppresses mammary tumorigenesis through regulation of the Hippo pathway. Oncogene 36, 2377-2393 (2017).

11. Cancino, G. I., Fatt, M. P., Miller, F. D. \& Kaplan, D. R. Conditional ablation of p63 indicates that it is essential for embryonic development of the central nervous system. Cell Cycle 14, 3270-3281 (2015).

12. Rouleau, M. et al. TAp63 is important for cardiac differentiation of embryonic stem cells and heart development. Stem Cells 29, 1672-1683 (2011).

13. Su, X. et al. TAp63 prevents premature aging by promoting adult stem cell maintenance. Cell. Stem Cell. 5, 64-75 (2009).

14. Raslan, A. A. \& Yoon, J. K. R-spondins: multi-mode WNT signaling regulators in adult stem cells. Int J. Biochem Cell Biol. 106, 26-34 (2019).

15. Carmon, K. S., Lin, Q., Gong, X., Thomas, A. \& Liu, Q. LGR5 interacts and cointernalizes with Wnt receptors to modulate Wnt/-catenin signaling. Mol. Cell Biol. 32, 2054-2064 (2012).

16. Shimokawa, M. et al. Visualization and targeting of LGR5+ human colon cancer stem cells. Nature 545, 187-192 (2017).

17. Shiokawa, D. et al. The induction of selected Wnt target genes by Tcf1 mediates generation of tumorigenic colon stem cells. Cell Rep. 19, 981-994 (2017).

18. Katoh, I. et al. Repression of Wnt/B-catenin response elements by p63 (TP63). Cell Cycle 15, 699-710 (2016).

19. Mori, N. et al. Cruciferous vegetable intake is inversely associated with lung cancer risk among current nonsmoking men in the Japan Public Health Center (JPHC) study. J. Nutr. 147, 841-849 (2017).

20. Gupta, P., Kim, B., Kim, S. \& Srivastava, S. K. Molecular targets of isothiocyanates in cancer: Recent advances. Mol. Nutr. Food Res. 58, 1685-1707 (2014).

21. Cheung, K. L. \& Kong, A. Molecular targets of dietary phenethyl isothiocyanate and sulforaphane for cancer chemoprevention. AAPS J. 12, 87-97 (2010).

22. Zhu, J. et al. miR-19 targeting of GSK3 $\beta$ mediates sulforaphane suppression of lung cancer stem cells. J. Nutr. Biochem. 44, 80-91 (2017).

23. Burnett, J. P. et al. Sulforaphane enhances the anticancer activity of taxanes against triple negative breast cancer by killing cancer stem cells. Cancer Lett. 394, 52-64 (2017).

24. Rausch, V. et al. Synergistic activity of sorafenib and sulforaphane abolishes pancreatic cancer stem cell characteristics. Cancer Res. 70, 5004-5013 (2010). 
25. Wang, Q. et al. The p53 family coordinates wnt and nodal inputs in mesendodermal differentiation of embryonic stem cells. Cell. Stem Cell. 20, 70-86 (2017).

26. Gatti, V. et al. p63 at the crossroads between stemness and metastasis in breast cancer. Int J. Mol. Sci. 20, 2683 (2019).

27. Li, B. et al. Detecting transcription factors with allosteric DNA-Silver nanocluster switches. Anal. Chim. Acta 1048, 168-177 (2019).

28. Gunaratne, P. H. et al. Activating p53 family member TAp63: a novel therapeutic strategy for targeting p53-altered tumors. Cancer-Am Cancer Soc 2409-2422 (2019).

29. Li, X. B. et al. Gastric Lgr5(+) stem cells are the cellular origin of invasive intestinal-type gastric cancer in mice. Cell Res. 26, 838-849 (2016).

30. Fujii, M. \& Sato, T. Defining the role of Lgr5+ stem cells in colorectal cancer: from basic research to clinical applications. Genome Med. 9, 66.https://doi.org/ 10.1186/s13073-017-0460-y (2017)

31. Keyes, W. M. et al. $\triangle \mathrm{Np63a}$ is an oncogene that targets chromatin remodeler Ish to drive skin stem cell proliferation and tumorigenesis. Cell. Stem Cell. 8 164-176 (2011)
32. Chen, $Y$. et al. Sulforaphane inhibits epithelial-mesenchymal transition by activating extracellular signal-regulated kinase 5 in lung cancer cells. J. Nutr. Biochem. 72, 108219 (2019).

33. Wang, $H$. et al. Pharmacokinetics and pharmacodynamics of phase II drug metabolizing/antioxidant enzymes gene response by anticancer agent sulforaphane in rat lymphocytes. Mol. Pharm. 9, 2819-2827 (2012)

34. Wang, D. X. et al. Sulforaphane suppresses EMT and metastasis in human lung cancer through miR-616-5p-mediated GSK3beta/beta-catenin signaling pathways. Acta Pharm. Sin. 38, 241-251 (2017).

35. Kelland, L. R. "Of mice and men": values and liabilities of the athymic nude mouse model in anticancer drug development. Eur. J. Cancer 40, 827-836 (2004).

36. Lee, N. P., Chan, C. M., Tung, L. N., Wang, H. K. \& Law, S. Tumor xenograft animal models for esophageal squamous cell carcinoma. J. Biomed. Sci. 25, 66, https://doi.org/10.1186/s12929-018-0468-7 (2018).

37. Day, C., Merlino, G. \& Van Dyke, T. Preclinical mouse cancer models: a maze of opportunities and challenges. Cell 163, 39-53 (2015).

38. Chen, Y. et al. Phenethyl isothiocyanate inhibits colorectal cancer stem cells by suppressing Wnt/ß-catenin pathway. Phytother. Res. 32, 2447-2455 (2018). 